\title{
Association of the Parathyroid Adenoma Volume and the Biochemical Parameters in Primary Hyperparathyroidism
}

\author{
Yul Hwang-Bo, Jung Hee Kim, Jee Hyun An, Eun Shil Hong, Jung Hun Ohn, Eun Ky Kim, Ah Reum Khang, \\ Sun Wook Cho, Do Joon Park, Kyong Soo Park, Seong Yeon Kim, Bo Youn Cho, Chan Soo Shin \\ Department of Internal Medicine, Seoul National University College of Medicine, Seoul, Korea
}

Background: The objective of this study is to demonstrate the relationship between the volume of a parathyroid adenoma and the preoperative biochemical parameters in patients undergoing surgery for primary hyperparathyroidism.

Methods: The medical records of 68 patients who underwent a parathyroidectomy for a single parathyroid adenoma were retrospectively reviewed. The volume of the adenoma was estimated using its measured size and a mathematical formula. The correlation between the volume of the parathyroid adenoma and the preoperative laboratory data was assessed.

Results: There were no correlations between the estimated volume of the adenoma and the serum calcium, alkaline phosphatase and parathyroid hormone levels. However, the volume of the adenoma was associated with the preoperative level of serum phosphorus. After excluding 5 adenomas with cystic degeneration, a positive correlation was noted between the adenoma volume and the preoperative levels of alkaline phosphatase and parathyroid hormone.

Conclusion: The preoperative serum levels of calcium, alkaline phosphatase and parathyroid hormone are of limited use to predict the volume of the parathyroid adenoma in patients with a single parathyroid adenoma. We suggest that the absence of a correlation between the volume of the adenoma and the biochemical parameters can be attributed to the cystic degeneration of the adenomas. (Endocrinol Metab 26:62-66, 2011)

Key Words: Hyperparathyroidism, Parathyroid adenoma, Parathyroidectomy

\section{INTRODUCTION}

Single parathyroid adenoma is the most common cause of primary hyperparathyroidism [1]. Primary hyperparathyroidism can be cured by surgical removal of the adenoma. Recently, on account of preoperatively imaging modality including technetium $99 \mathrm{~m}$ sestamibi scintigraphy and ultrasonography, minimally invasive procedures are becoming increasingly common [2]. The measurement of the intraoperative parathyroid hormone level also helps localize an adenoma $[3,4]$. Despite the advancement of localizing techniques, none of the currently available modalities has reached perfection in terms of an ability to localize an adenoma.

The relationship between the size of an adenoma and the biochemical parameters such as serum calcium, phosphorus and parathyroid hormone in a case of primary hyperparathyroidism is unclear. If a relationship exists between the size of the adenoma

Received: 27 July 2010, Accepted: 28 September 2010

Corresponding author: Chan Soo Shin

Department of Internal Medicine, Seoul National University Hospital,

101 Daehak-ro, Jongno-gu, Seoul 110-744, Korea

Tel: +82-2-2072-3734, Fax: +82-2-762-9662,E-mail: csshin@snu.ac.kr and the biochemical parameters, radiologist and surgeon could get additional information predicting size of the adenoma by biochemical parameters. And formulating a sense of size of the functioning adenoma by biochemical parameters helps to localize adenoma in case of multiple nodules in neck sonography.

According to previous studies, the relationship between preoperative laboratory parameters and the size of an adenoma could vary. In 1987, Dubost et al. [5] reported that the parathyroid hormone level was not correlated with the weight of the adenoma in patients with primary hyperparathyroidism. However, several papers in the 1980s reported that the volume or weight of the adenoma was significantly correlated with the serum calcium and parathyroid hormone levels [6-8]. In 1995, Hedback et al. [9] reported that the adenoma weight was significantly related to the risk of death in 713 patients with a single parathyroid gland disease. This group also found a significant correlation between the glandular weight and the preoperative serum calcium level. Recently, Randhawa et al. [10] concluded that there was no correlation between preoperative biochemical markers of calcium homeostasis and the adenoma weight or volume. Other researchers also re- 
ported similar results [11-13]. To date, data from various studies show conflicting result.

Parathyroid adenomas could have various pathologic features. In some patients, primary hyperparathyroidism spontaneously remits through parathyroid apoplexy [14,15]. McCoy et al. [16] found that cystic parathyroid lesions were common in the pathologic findings of primary hyperparathyroidism. These pathologic changes can have an effect on laboratory parameters. To our knowledge, there was no study to evaluate relationship of parathyroid adenoma volume and biochemical parameters with consideration of pathologic findings. In this study, we aimed to identify laboratory parameters that predict the size of a parathyroid adenoma while considering pathologic changes in patients with primary hyperparathyroidism.

\section{METHODS}

\section{Subjects}

From January of 2000 to May of 2008, 131 patients were diagnosed with primary hyperparathyroidism at Seoul National University Hospital. A retrospective review of hospital records of these patients was performed. Among these patients, 36 were not indicated as having undergone a parathyroidectomy, having been treated without surgery. Two patients underwent radiofrequency ablation due to concomitant medical conditions. Ninety three patients were operated on at the same hospital. Among the patients who underwent surgery, patients with double adenoma $(n=1)$, hyperplasia $(n=6)$, carcinoma $(n=6)$, hypercalcemic crisis upon admission (serum calcium level greater than $15 \mathrm{mg} / \mathrm{dL} ; \mathrm{n}=4$ ) [17], chronic renal failure (serum creatinine level greater than $1.5 \mathrm{mg} /$ $\mathrm{dL} ; \mathrm{n}=5$ ), active malignancy $(\mathrm{n}=2)$, and multiple endocrine neoplasia type $1(n=1)$ were excluded. The data from 68 patients who were diagnosed with single parathyroid adenoma after surgery were analyzed.

\section{Methods}

Preoperative laboratory data including serum calcium, phosphorus, alkaline phosphatase, and parathyroid hormone levels were used in the analysis. Serum parathyroid hormone was measured preoperatively by means of immunoradiometric assay (Cis Bio International, Gif-Sur-Yvette, Cedex, France). The excised parathyroid adenomas were evaluated by an experienced pathologist. The size of the parathyroid adenoma was measured. The volume of the adenomas was calculated using the mathematical formula shown be- low for the volume of an ellipsoid object: estimated volume $=\pi / 6$ $\times \mathrm{a} \times \mathrm{b} \times \mathrm{c}$ (a: the major axis, b: the minor axis, c: height, $\pi=$ 3.14159) [18-20]. In a gross pathology examination, the pathologist described presence of necrosis, cystic change and hemorrhage of the parathyroid adenoma.

\section{Statistical analysis}

Statistical analyses were carried out using SPSS 16.0 for Windows (SPSS, Chicago, IL, USA). Statistical analysis was performed with the goal of demonstrating the relationship between the estimated volume of the parathyroid adenoma and the preoperative biochemical parameters. Data were expressed as the mean \pm standard deviation. For correlation studies, Pearson's correlation coefficients (R) were applied. Statistical significance was inferred with $P$ values $<$ 0.05 .

\section{RESULTS}

Among the 68 patients, 26 patients were men and 42 were women. The mean age of the patients was $51.2 \pm 15.9$ years (range 15-84). Twenty five (37\%) patients had history of renal stone disease, 18 (26\%) patients had osteoporosis, and 3 (4\%) patients had osteoporotic fracture. Forty seven patients have serum calcium levels that are more than $1 \mathrm{mg} / \mathrm{dL}$ above the upper limits of normal $(10.5 \mathrm{mg} /$ dL). Fourteen patients underwent bilateral neck explorations, and 50 patients underwent unilateral neck explorations. Four patients underwent a minimally invasive parathyroidectomy. Mean serum biochemical parameters values were as follows: serum calcium $=$ $11.50 \pm 0.75 \mathrm{mg} / \mathrm{dL}$, serum phosphorus $=2.50 \pm 0.45 \mathrm{mg} / \mathrm{dL}$, serum alkaline phosphatase $=115.9 \pm 49.8 \mathrm{IU} / \mathrm{L}$, and serum parathyroid

Table 1. Characteristics of primary hyperparathyroidism in patients of single parathyroid adenoma

\begin{tabular}{lcc}
\hline & & Normal range \\
\hline Number & 68 & \\
Sex (male/female) & $26 / 42$ & \\
Age (years) & $51.2 \pm 15.9$ & \\
Volume of adenoma (mm²) & $908.6-1178.8$ & \\
Serum calcium (mg/dL) & $11.50 \pm 0.75$ & $8.8-10.5$ \\
Serum phosphorus (mg/dL) & $2.50 \pm 0.45$ & $2.5-4.5$ \\
Alkaline phosphatase (IU/L) & $115.9 \pm 49.8$ & $30-115$ \\
Parathyroid hormone $(\mathrm{pg} / \mathrm{mL})$ & $168.1 \pm 117.4$ & $10-65$ \\
eGFR (mL/min/1.73 m²) & $79.6 \pm 16.6$ & \\
\hline
\end{tabular}

eGFR, estimate of glomerular filtration rate (by Modification of Diet in Renal Disease Study equation). 
hormone level $=168.1 \pm 117.4 \mathrm{pg} / \mathrm{mL}$ (Table 1 ).

In the gross pathology examination, 4 adenomas had cystic lesions, 7 showed hemorrhage, 1 had necrotic changes, and 1 had both a cystic lesion and hemorrhage. Adenomas that had the highest $\left(6963 \mathrm{~mm}^{3}\right)$ and second highest $\left(5498 \mathrm{~mm}^{3}\right)$ values of the calculated volume showed cystic change in the central portion of the adenoma.

We compared of the adenoma volume and biochemical parameters according to the presence of cystic change. There were no differences between adenomas with cystic change and those without cystic change with respect to the biochemical parameters. The size of an adenoma with cystic change was larger than that without cystic change (Table 2).

The volume of the adenoma was not correlated with the serum calcium, alkaline phosphatase or parathyroid hormone levels. Only the serum phosphorus level was significantly correlated with the estimated adenoma volume $(P=0.048)$. After the exclusion of 5 adenoma that showed cystic change, the adenoma volume correlated with the preoperative serum alkaline phosphatase $(R=0.269$, $P=0.033)$ and parathyroid hormone $(R=0.274, P=0.030)$ levels. However there were no correlations between the volume of the adenoma and the serum phosphorus or calcium levels. After the exclusion of 7 adenomas with hemorrhage, no correlation existed between the parathyroid adenoma volume and biochemical parameters (data are not shown). After the exclusion of 13 adenoma with cystic lesions, hemorrhage or necrosis, an analysis showed a correlation between the volume of the adenoma and the alkaline

Table 2. Comparison of adenoma volume and biochemical parameters according to presence of cystic change

\begin{tabular}{lccc}
\hline & $\begin{array}{c}\text { Adenoma with } \\
\text { cystic change } \\
(\mathrm{n}=5)\end{array}$ & $\begin{array}{c}\text { Adenoma without } \\
\text { cystic change } \\
(\mathrm{n}=63)\end{array}$ & $P$ \\
\hline Volume of adenoma $\left(\mathrm{mm}^{3}\right)$ & $3330.2 \pm 2829.3$ & $706.1 \pm 940.2$ & 0.005 \\
\hline Serum calcium $(\mathrm{mg} / \mathrm{dL})$ & $11.2 \pm 0.31$ & $11.6 \pm 0.77$ & 0.130 \\
\hline Serum phosphorus $(\mathrm{mg} / \mathrm{dL})$ & $2.4 \pm 0.36$ & $2.5 \pm 0.46$ & 0.615 \\
\hline Alkaline phosphatase $(\mathrm{IU} / \mathrm{L})$ & $103.6 \pm 48.8$ & $116.9 \pm 50.2$ & 0.568 \\
\hline Parathyroid hormone $(\mathrm{pg} / \mathrm{mL})$ & $209.4 \pm 84.8$ & $164.8 \pm 119.5$ & 0.156 \\
\hline
\end{tabular}

phosphatase $(R=0.275, P=0.042)$ and parathyroid hormone levels $(R=0.301, P=0.026)$ (Table 3).

\section{DISCUSSION}

In this study, we hypothesized that preoperative biochemical parameters would have a predictive value regarding the size of the adenoma. However, there was no correlation between the volume of the adenoma and the serum calcium, parathyroid hormone, or alkaline phosphatase levels. Previous studies attempted to demonstrate a relationship between the size of the adenoma and various biochemical parameters (Table 4). In the majority of earlier studies, there was an association between the parathyroid hormone level and the volume or weight of the parathyroid adenoma [6-8,13,18, 21,22]. A positive correlation between the serum calcium level and either the adenoma weight or volume has also been reported [6$9,18,22]$. However, other studies have not shown any correlation between these variables $[5,10-12,23]$. In this study, we could not

Table 4. Previous studies about relationship between adenoma size and preoperative parameters

\begin{tabular}{lrrrrrrr}
\hline & N & Age & Ca & PTH & ALP & P & Cl/P \\
\hline Dubost et al. (1978) [5] & 52 & & & X & & & \\
Wagner et al. (1983) [23] & 48 & & X & X & X & X & \\
Rutledge et al. (1985) [6] & 92 & & 0 & 0 & & & \\
Zamboni et al. (1986) [7] & 82 & & 0 & 0 & & & \\
Saxe et al. (1987) [8] & 41 & & 0 & 0 & & & \\
Saadeh et al. (1989) [11] & 24 & & X & X & & & \\
Williams et al. (1992) [12] & 44 & & X & X & & & \\
Hedback et al. (1995) [9] & 713 & & 0 & & & & \\
Bindlish et al. (2002) [18] & 63 & & 0 & 0 & & $X$ & \\
Mozes et al. (2002) [21] & 166 & & X & 0 & & & \\
Biertho et al. (2004) [28] & 220 & X & X & 0 & X & & \\
Turgut et al. (2006) [13] & 40 & 0 & X & X & 0 & & \\
Randhawa et al. (2007) [10] & 77 & & X & X & & & \\
Calva-Cerqueira et al. & 198 & & 0 & 0 & 0 & & 0 \\
(2007) [22] & & & & & & & \\
\hline
\end{tabular}

$\mathrm{N}$, number; Ca, serum calcium; PTH, parathyroid hormone; ALP, alkaline phosphatase; $\mathrm{P}$, serum phosphorus; $\mathrm{CI} / \mathrm{P}$, chloride: phosphorus ratio; $\mathrm{O}$, correlation exist; $X$, no correlation exist.

Table 3. Correlation of calculated volume of excised parathyroid adenoma with preoperative biochemical parameters

\begin{tabular}{llll} 
& \multicolumn{1}{c}{ All $(\mathrm{n}=68)$} & Adenoma without cystic change $(\mathrm{n}=63)$ & $\begin{array}{c}\text { Adenoma without cystic change, } \\
\text { hemorrhage and necrosis }(\mathrm{n}=55)\end{array}$ \\
\hline Serum calcium & $R=-0.037, P=0.765$ & $R=-0.044, P=0.730$ & $R=0.024, P=0.863$ \\
Serum phosphorus & $R=-0.241, P=0.048$ & $R=-0.215, P=0.090$ & $R=-0.196, P=0.151$ \\
Alkaline phosphatase & $R=0.047, P=0.706$ & $R=0.269, P=0.033$ & $R=0.275, P=0.042$ \\
Parathyroid hormone & $R=0.155, P=0.208$ & $R=0.274, P=0.030$ & $R=0.301, P=0.026$ \\
\hline
\end{tabular}


demonstrate a correlation between the volume of the adenoma and either the level of intact parathyroid hormone or serum calcium. Thus, preoperative serum markers are unreliable to predict the adenoma volume. Our data showed a significant correlation between the serum phosphorus level and the adenoma volume, contradicting a number of earlier studies [18,23]. Further studies are warranted to confirm our observation.

In this study, 5 adenomas showed cystic lesions (4 adenomas; cystic lesion only, 1 adenoma; cystic change and hemorrhage). The incidence of cystic parathyroid adenoma (7.4\%) was higher than that found in a large previous study (3\%) [16]. The cystic adenoma was larger than the adenoma without cystic change. However, there was no difference in the biochemical parameters in both groups. When 5 cystic adenomas were removed from the analysis, the correlation between the volume of the adenoma and the levels of alkaline phosphatase and parathyroid hormone were significant. However, the association between the level of serum phosphorus and the adenoma volume disappeared. Thus, our results indicate that biochemical parameters could be affected by pathology of adenoma. The cystic change of adenoma is considered as a major determinant of biochemical markers in large sized adenoma.

In spite of the exclusion of confounding variables such as the existence of renal failure, data from earlier studies did not reach a consensus regarding a relationship between laboratory tests and adenoma volume. Various explanations are possible in relation to this discordance. One explanation for these contradictory results may be the pathologic differences between the adenomas in each study. A smaller proportion of parathyroid hormone secreting cells in a larger adenoma could result from a greater proportion of the adenoma being composed of cystic change or hemorrhage [12]. If cases of a study include many adenomas with pathologic changes such as cystic formation, necrosis or hemorrhage, a correlation between the volume of the adenoma and biochemical parameters would not exist. In most previous studies, the specific pathologic features of the adenomas were not described. In this study, we suggest that cystic change of the parathyroid adenoma is an important pathologic feature that causes discrepancies between biochemical parameters and the volume of the adenoma.

The etiology of parathyroid cyst formation remains a question. Various theories have been proposed [24]. Functioning parathyroid cyst may result from degeneration of a parathyroid adenoma. Another theory suggests that parathyroid cysts originate from an accumulation of secretions with a gradual enlargement of the cyst
[25]. A parathyroid cyst contains a relatively small portion of parenchyma in its volume. Thus, we inferred that parathyroid cysts release relatively small amounts of hormone. In this study, an association between biochemical parameters and the adenoma volume could be attenuated by the relatively high proportion of cystic parathyroid adenomas.

Among five adenomas with cystic change in their pathology, four adenomas showed cyst features in the neck ultrasonography results. This finding may have practical implications for patients with cystic parathyroid lesions as noted by ultrasonography. In patients with hyperparathyroidism who show a cystic lesion in their neck ultrasonography, a discrepancy between laboratory results such as the parathyroid hormone level and the size according to the ultrasonography results is possible because cystic adenomas have relatively low levels of parathyroid hormone in its volume.

In the present study, intracapsular hemorrhage was observed in eight adenomas. Kataoka et al. [26] reported a case with a recurrence of primary hyperparathyroidism 4 months after spontaneous remission with acute intracapsular hemorrhage of a parathyroid adenoma. Wootten et al. [27] summarized cases of spontaneous remission of primary hyperparathyroidism in the literature. Most of the cases were accompanied with acute clinical aberrations and laboratory changes. The pathologic changes, including autoinfarction, acute extracapsular hemorrhage, and acute intracapsular hemorrhage in these cases appear to be acute events. The hemorrhagic change of the adenoma in our data may represent a chronic event due to the absence of laboratory aberrations. The clinical implications of intracapsular hemorrhage are not definite.

To the best of our knowledge, this is the first study to report a relationship between biochemical parameters and adenoma volume considering pathologic changes such as cystic change and hemorrhage. This study also had a large sample size.

In summary, our data suggest that biochemical parameters have limited usefulness when estimating the volume of a parathyroid adenoma. The absence of a correlation between the volume of an adenoma and the biochemical parameters is attributed to cystic change in adenomas.

\section{REFERENCES}

1. Kebebew E, Hwang J, Reiff E, Duh QY, Clark OH: Predictors of singlegland vs multigland parathyroid disease in primary hyperparathyroidism: a simple and accurate scoring model. Arch Surg 141:777-782; 2006

2. Mariani G, Gulec SA, Rubello D, Boni G, Puccini M, Pelizzo MR, Manca 
G, Casara D, Sotti G, Erba P, Volterrani D, Giuliano AE: Preoperative localization and radioguided parathyroid surgery. J Nucl Med 44:1443-1458, 2003

3. Irvin GL 3rd: American Association of Endocrine Surgeons. Presidential address: chasin' hormones. Surgery 126:993-997, 1999

4. Irvin GL 3rd, Carneiro DM, Solorzano CC: Progress in the operative management of sporadic primary hyperparathyroidism over 34 years. Ann Surg 239:704-708, 704-711, 2004

5. Dubost C, Bordier PJ, Ferry J, Gueris J: (The estimation of parathormone in primary hyperparathyroidism). Nouv Presse Med 7:21-25, 1978

6. Rutledge R, Stiegel M, Thomas CG Jr, Wild RE: The relation of serum calcium and immunoparathormone levels to parathyroid size and weight in primary hyperparathyroidism. Surgery 98:1107-1112, 1985

7. Zamboni WA, Folse R: Adenoma weight: a predictor of transient hypocalcemia after parathyroidectomy. Am J Surg 152:611-615, 1986

8. Saxe AW, Lincenberg S, Hamburger SW: Can the volume of abnormal parathyroid tissue be predicted by preoperative biochemical measurement? Surgery 102:840-845, 1987

9. Hedback G, Oden A, Tisell LE: Parathyroid adenoma weight and the risk of death after treatment for primary hyperparathyroidism. Surgery 117 : 134-139, 1995

10. Randhawa PS, Mace AD, Nouraei SA, Stearns MP: Primary hyperparathyroidism: do perioperative biochemical variables correlate with parathyroid adenoma weight or volume? Clin Otolaryngol 32:179-184, 2007

11. Saadeh G, Licata A, Esselstyn C, Gupta M: Relationship of parathyroid adenoma volume and biochemical function. Horm Res 32:142-144, 1989

12. Williams JG, Wheeler MH, Aston JP, Brown RC, Woodhead JS: The relationship between adenoma weight and intact (1-84) parathyroid hormone level in primary hyperparathyroidism. Am J Surg 163:301-304, 1992

13. Turgut B, Elagoz S, Erselcan T, Koyuncu A, Dokmetas HS, Hasbek Z, Ozdemir S, Aydin C: Preoperative localization of parathyroid adenomas with technetium-99m methoxyisobutylisonitrile imaging: relationship with P-glycoprotein expression, oxyphilic cell content, and tumoral tissue volume. Cancer Biother Radiopharm 21:579-590, 2006

14. Nylen E, Shah A, Hall J: Spontaneous remission of primary hyperparathyroidism from parathyroid apoplexy. J Clin Endocrinol Metab 81:1326-1328, 1996

15. Kovacs KA, Gay JD: Remission of primary hyperparathyroidism due to spontaneous infarction of a parathyroid adenoma. Case report and review of the literature. Medicine (Baltimore) 77:398-402, 1998
16. McCoy KL, Yim JH, Zuckerbraun BS, Ogilvie JB, Peel RL, Carty SE: Cystic parathyroid lesions: functional and nonfunctional parathyroid cysts. Arch Surg 144:52-56, 2009

17. Lew JI, Solorzano CC, Irvin GL 3rd: Long-term results of parathyroidectomy for hypercalcemic crisis. Arch Surg 141:696-699, 2006

18. Bindlish V, Freeman JL, Witterick IJ, Asa SL: Correlation of biochemical parameters with single parathyroid adenoma weight and volume. Head Neck 24:1000-1003, 2002

19. Brasier AR, Nussbaum SR: Hungry bone syndrome: clinical and biochemical predictors of its occurrence after parathyroid surgery. Am J Med 84:654-660, 1988

20. Piga M, Bolasco P, Satta L, Altieri P, Loi G, Nicolosi A, Tarquini A, Mariotti S: Double phase parathyroid technetium-99m-MIBI scintigraphy to identify functional autonomy in secondary hyperparathyroidism. J Nucl Med 37:565-569, 1996

21. Mozes G, Curlee KJ, Rowland CM, van Heerden JA, Thompson GB, Grant CS, Farley DR: The predictive value of laboratory findings in patients with primary hyperparathyroidism. J Am Coll Surg 194:126-130, 2002

22. Calva-Cerqueira D, Smith BJ, Hostetler ML, Lal G, Menda Y, O’Dorisio TM, Howe JR: Minimally invasive parathyroidectomy and preoperative MIBI scans: correlation of gland weight and preoperative PTH. J Am Coll Surg 205(4 Suppl):S38-44, 2007

23. Wagner PK, Rothmund M: Correlation of tumor weight and typical pathologic laboratory parameters in primary and secondary hyperparathyroidism. Langenbecks Arch Chir 360:133-139, 1983

24. Safran D: Functioning parathyroid cyst. South Med J 91:978-980, 1998

25. Fortson JK, Patel VG, Henderson VJ: Parathyroid cysts: a case report and review of the literature. Laryngoscope 111:1726-1728, 2001

26. Kataoka K, Taguchi M, Takeshita A, Miyakawa M, Takeuchi Y: Recurrence of primary hyperparathyroidism following spontaneous remission with intracapsular hemorrhage of a parathyroid adenoma. J Bone Miner Metab 26: 295-297, 2008

27. Wootten CT, Orzeck EA: Spontaneous remission of primary hyperparathyroidism: a case report and meta-analysis of the literature. Head Neck 28: 81-88, 2006

28. Biertho LD, Kim C, Wu HS, Unger P, Inabnet WB: Relationship between sestamibi uptake, parathyroid hormone assay, and nuclear morphology in primary hyperparathyroidism. J Am Coll Surg 199:229-233, 2004 